\title{
Vector mesons at finite density from QCD sum rules and the maximum entropy method
}

Philipp Gubler*

RIKEN, Nishina Center, Hirosawa 2-1, Wako, Saitama, 351-0198, Japan

E-mail: pgublereriken.jp

\section{Keisuke Ohtani}

Tokyo Institute of Technology, Meguro 2-12-1, Tokyo 152-8551, Japan

E-mail: ohtani.keth.phys.titech.ac.jp

\begin{abstract}
The modification of vector mesons at finite density is studied by using QCD sum rules in combination with the maximum entropy method. As a result, it is found that the $\rho$ and $\omega$ receive a negative mass shift of about $100 \mathrm{MeV}$ at nuclear matter density, which is consistent with previous studies. This finding however strongly depends of the factorization assumption of the four-quark condensate, which might not be quantitatively reliable. On the other hand, we find that the mass shift of the $\phi$ is smaller, but much less dependent on the four quark condensate. Instead, our results show that the $\phi$ mass shift is strongly correlated to the strangeness content of the nucleon, which governs the depletion of the strange quark condensate in nuclear matter.
\end{abstract}

XV International Conference on Hadron Spectroscopy-Hadron 2013

4-8 November 2013

Nara, Japan

${ }^{*}$ Speaker. 


\section{Introduction}

Light vector mesons at finite density have been studied already for quite a long time. They are interesting especially because the vector mesons have the potential to carry information on the partial restoration of chiral symmetry in nuclear matter, which could be measured in experiments [1]. Early works studying QCD sum rules for the vector channels at finite density [1] 3] therefore provided strong incentives for later experiments.

Matters have however turned out to be a bit more complicated than they were thought to be. Even though QCD sum rules indeed provide some relation between the spectral function of the various vector channels and the order parameters of chiral symmetry, this relation is not a direct one for the $\rho$ and $\omega$ as the driving term for the modification of the spectrum entering into the sum rules contains not the most simple two-quark condensate but the less known four-quark condensate. Furthermore, the change of the spectral function should not be considered to be a simple mass shift of the ground state peak, but rather a combination of mass shift and some sort of broadening for which the sum rules give only a weak constraint [4]. For the $\phi$, these issues are less severe because the sum rule input depends much less on the four-quark condensate and its broadening at nuclear matter density has been estimated to be well below $100 \mathrm{MeV}$ [5].

In our work to be summarized in these proceedings, we analyze the vector meson sum rules in vacuum and at finite density in a more general way than it was done before, namely with the help of a recently developed approach which makes use of the maximum entropy method (MEM) [6]. This method makes it possible to obtain the most likely spectral function directly from the operator product expansion (OPE), without having to make strong assumptions on its functional form. It is particularly useful for studying the modification of spectral functions at finite density, as the framework used to study the vacuum spectral function can be applied analogously to the nuclear matter case.

This article is organized as follows. In section 2.1 we will first briefly recapitulate the basics of QCD sum rules and its application to finite density. Next, the OPE of the various channels will be discussed in section 2.2 and the corresponding analysis results presented in section 3 Finally, the conclusions are given in section 4

\section{Formalism}

\subsection{Basics of QCD sum rules}

As it is common when working with QCD sum rules, we start with the two-point function of an interpolating field coupling strongly to the hadron of interest:

$$
\Pi_{\mu v}(\omega, \vec{q})=i \int d^{4} e^{i q x}\left\langle\mathrm{~T}\left[j_{\mu}(x) j_{v}(0)\right]\right\rangle_{\rho} .
$$

The operator $j_{\mu}$ here stands for $\frac{1}{2}\left(\bar{u} \gamma_{\mu} u \mp \bar{d} \gamma_{\mu} d\right)$, in which the negative (positive) sign is used for the $\rho(\omega)$ and for $\bar{s} \gamma_{\mu} s$ for the $\phi .\langle\rangle_{\rho}$ represents the expectation value with respect to the ground state of nuclear matter. 
We will in the following only consider mesons at rest relative to the nuclear medium $(\vec{q}=0)$ and therefore only need to study the contracted correlator defined as

$$
\Pi\left(\omega^{2}\right)=-\frac{1}{3 \omega^{2}} \Pi_{\mu}^{\mu}(\omega, \vec{q}=0) .
$$

From the analytic properties of $\Pi\left(\omega^{2}\right)$, one can then derive the following dispersion relation

$$
\Pi\left(\omega^{2}\right)=\frac{1}{\pi} \int_{0}^{\infty} d s \frac{\operatorname{Im} \Pi(s)}{s-\omega^{2}-i \varepsilon} .
$$

As a next step, one takes $\omega^{2}$ as a large and negative number and calculates the left-hand side of Eq.(2.3) using the OPE. This gives a power series in $1 / \omega^{2}$ with the Wilson-coefficients expressed as expansions in the strong coupling constant $\alpha_{s}$. On the right hand side, the function $\frac{1}{\pi} \operatorname{Im} \Pi(s)$ is considered in terms of hadronic degrees of freedom and hence contains information on the phyiscal states coupling to $j_{\mu}(x)$.

As a last step, the Borel transform, which cancels any subtraction constants, renders the integral over $s$ convergent and furthermore improves the convergence of the OPE, is applied, giving

$$
\tilde{\Pi}\left(M^{2}\right)=\frac{2}{M^{2}} \int_{0}^{\infty} d \omega \omega e^{-\omega^{2} / M^{2}} \rho(\omega),
$$

where the spectral function $\rho(\omega)$ has been defined as $\rho(\omega)=\frac{1}{\pi} \operatorname{Im} \Pi\left(\omega^{2}\right)$. This is the form of the sum rule that will be employed presented in these proceedings. For its analysis, we will use MEM, by which we can extract the most probable form of $\rho(\omega)$ directly from $\tilde{\Pi}\left(M^{2}\right)$. The details of this analysis method can be found for instance in [6, $7,8,9$.

\subsection{The OPE of the vector channels in the linear density approximation}

The OPE can generally be given as shown below:

$$
\Pi_{O P E}\left(M^{2}, \rho\right)=c_{0}(\rho)+\frac{c_{1}(\rho)}{M^{2}}+\frac{c_{2}(\rho)}{M^{4}}+\frac{c_{3}(\rho)}{M^{6}}+\ldots
$$

Here, we consider terms up to dimension 6 and hence neglect all terms not explicitly shown above. The coefficients $c_{i}(\rho)$ contain dimensionless functions and condensates of mass dimension $2 i$.

In vacuum $(\rho=0)$, the $c_{i}(0)$ 's have been obtained as [10]

$$
\begin{aligned}
c_{0}(0) & =\frac{1}{4 \pi^{2}}\left(1+\frac{\alpha_{s}}{\pi}\right), & c_{2}(0) & =\frac{1}{12}\left\langle\frac{\alpha_{s}}{\pi} G^{2}\right\rangle+2 m_{q}\langle\bar{q} q\rangle, \\
c_{1}(0) & =-\frac{3 m_{q}^{2}}{2 \pi^{2}}, & c_{3}(0) & =-\frac{112}{81} \pi \alpha_{s} \kappa\langle\bar{q} q\rangle^{2},
\end{aligned}
$$

for the $\rho$ and $\omega$ mesons. In the above expressions, quantities labeled as $q$ are averaged over the $u$ and $d$ quarks. Within our approximations, both $\rho$ and $\omega$ give the same OPE. We will therefore from now on only refer to the $\rho$, the same results also being applicable to the $\omega$ meson. The OPE for the $\phi$ meson can be similarly obtained by exchanging $m_{q}$ with $m_{s},\langle\bar{q} q\rangle$ with $\langle\bar{s} s\rangle$ and the factor $\frac{112}{81}$ at dimension 6 with $\frac{80}{81}$. The main difference between the two cases arises from the term proportional to the quark masses at dimension 2 and 4 . For the $\rho$, these terms are essentially negligible, while they have important effects in the $\phi$ meson case. 

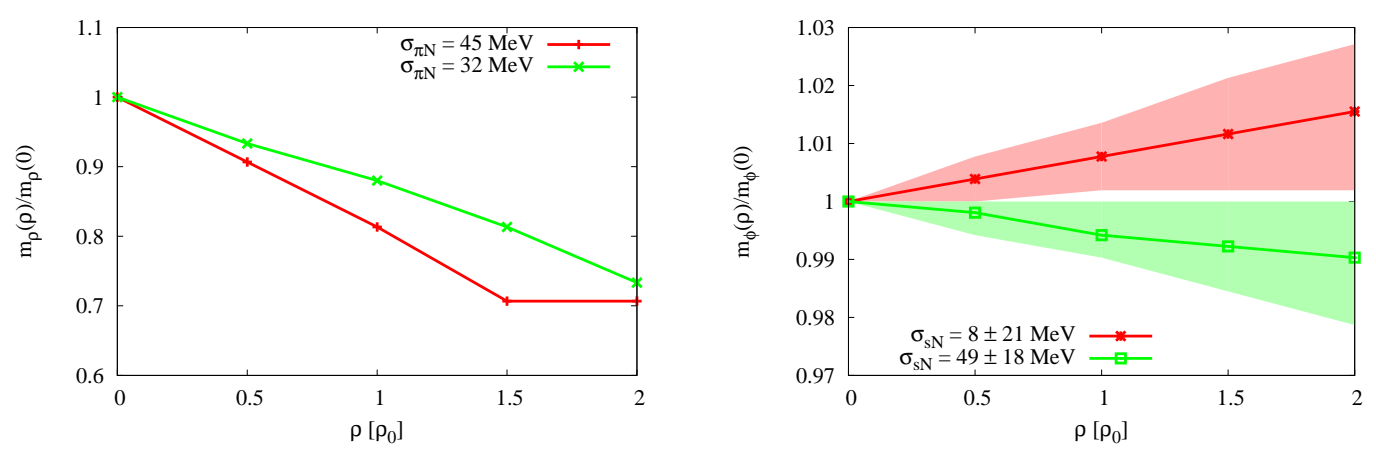

Figure 1: The $\rho$ (left plot) and $\phi$ (right plot) meson masses as a function of density. The masses are given relative to their vacuum values, while the density is given in units of the nuclear matter density $\rho_{0}$.

Next, let us proceed to the OPE at finite density. Within the linear density approximation, the coefficients $c_{i}(\rho)$ can be given as [1]

$$
\begin{array}{ll}
c_{0}(\rho)=c_{0}(0), & c_{2}(\rho)=c_{2}(0)+\rho\left[-\frac{2}{27} M_{N}^{0}+2 m_{q}\langle N|\bar{q} q| N\rangle+\frac{1}{2} A_{1}^{u+d} M_{N}\right] \\
c_{1}(\rho)=c_{1}(0), & c_{3}(\rho)=c_{3}(0)+\rho\left[-\frac{224}{81} \pi \alpha_{s} \kappa\langle\bar{q} q\rangle\langle N|\bar{q} q| N\rangle .-\frac{5}{12} A_{3}^{u+d} M_{N}^{3}\right],
\end{array}
$$

for the $\rho$ meson case, while for the $\phi$ we get

$$
\begin{array}{ll}
c_{0}(\rho)=c_{0}(0), & c_{2}(\rho)=c_{2}(0)+\rho\left[-\frac{2}{27} M_{N}^{0}+2 m_{s}\langle N|\bar{s} s| N\rangle+A_{1}^{s} M_{N}\right], \\
c_{1}(\rho)=c_{1}(0), & c_{3}(\rho)=c_{3}(0)+\rho\left[-\frac{160}{81} \pi \alpha_{s} \kappa\langle\bar{s}\rangle\langle N|\bar{s} s| N\rangle \cdot-\frac{5}{6} A_{3}^{s} M_{N}^{3}\right] .
\end{array}
$$

Here, $M_{N}$ is the nucleon mass and $M_{N}^{0}$ its value at the chiral limit. Furthermore, the variables $A_{1}^{u+d}$, $A_{3}^{u+d}, A_{1}^{s}$ and $A_{3}^{s}$ are moments over the parton distributions of the $u+d$ and $s$ quarks, respectively.

\section{Results}

Let us discuss our obtained results on the behavior of the various vector mesons at finite density. Due to limitation of space, we here will only show our findings on the meson mass shifts. Detailed discussions taking into account the effects of broadening and and other systematic uncertainties will be given elsewhere [11].

Firstly, the $\rho$-mass is shown as a function of density on the left side of Fig. 1 As can be observed in this figure, the $\rho$ meson experiences a negative mass shift, which takes a value of about $100 \mathrm{MeV}$ at nuclear matter density, the detailed number depending somewhat on the used value of the $\pi N$ sigma term: $\sigma_{\pi N}=2 m_{q}\langle N|\bar{q} q| N\rangle$. This result is essentially in agreement with earlier 
works [1]. We should however add here some comment of caution, as the vacuum saturation approximation used in Eqs. (2.7) and 2.9), might be strongly violated, especially when going to finite density. As the four-quark condensate term in mainly responsible for the OPE modification at finite density, this approximation introduces large uncertainties into our results for the $\rho$ meson.

For the $\phi$ meson, the situation is much better because in the OPE of this channel, it is mainly the dimension 4 terms, which determine the change of the OPE at finite density. The most important unknown quantity of this sum rule is in fact the strange sigma term $\sigma_{s N}=m_{s}\langle N|\bar{s} s| N\rangle$, which is illustrated on the right side of Fig. 1, where the $\phi$ meson mass is shown as a function of density for two typical values of $\sigma_{s N}$, which have been obtained by two recent lattice QCD calculations [12]13]. It should also be noted, that the mass shifts that we have obtained here are much smaller than the ones extracted in earlier works [1], one of them even having a different sign. The reason for this discrepancy lies in the recent values of $\sigma_{s N}$, which are up to a factor of five smaller than the estimates used in [1].

\section{Conclusions}

We have studied the behavior of vector mesons at finite density using QCD sum rules and MEM. As a result, we have found a negative mass shift of about $100 \mathrm{MeV}$ for the $\rho$ and $\omega$ mesons at nuclear matter density, which agrees with earlier estimates. This result involves, however large uncertainties due to our lack of knowledge on the behavior of the four-quark condensates at finite density.

For the $\phi$ meson, we have found a strong dependence of the finite density mass shift on the strange sigma term, which in the future might make it possible to constrain this quantity by experimentally measuring the $\phi$ meson mass shift. The details of this last point will be discussed in detail in a forthcoming publication [11].

\section{References}

[1] T. Hatsuda and S.H. Lee, Phys. Rev. C 46, 34 (1992).

[2] S. Leupold, V. Metag and U. Mosel, Int. J. Mod. Phys. E 19, 147 (2010).

[3] F. Klingl, N. Kaiser and W. Weise, Nucl. Phys. A624, 527 (1997).

[4] S. Leupold, W. Peters and U. Mosel, Nucl. Phys. A628, 311 (1998).

[5] A. Polyanskiy et al., Phys. Lett. B 695, 74 (2011).

[6] P. Gubler and M. Oka, Prog. Thor. Phys. 124, 995 (2010).

[7] P. Gubler, A Bayesian Analysis of QCD Sum Rules, Springer Theses, Springer Japan, 2013.

[8] M. Jarrel and J.E. Gubernatis, Phys. Rep. 269, 133 (1996).

[9] M. Asakawa, T. Hatsuda, and Y. Nakahara, Prog. Part. Nucl. Phys. 46, 459 (2001).

[10] M.A. Shifman, A.I. Vainshtein and V.I. Zakharov, Nucl. Phys. B147, 385 (1979); B147, 448 (1979).

[11] P. Gubler and K. Ohtani, in preparation.

[12] H. Ohki et al. (JLQCD Collaboration), Phys. Rev. D 87, 034509 (2013).

[13] P. Junnarkar and A. Walker-Loud, Phys. Rev. D 87, 114510 (2013). 